\title{
Green Area in Urban Cuiabá: The Case of the Universidade Federal do Mato Grosso
}

\author{
Poliane Pierra Patricio Pazini ${ }^{1}$ (1) 0000-0002-5924-809X \\ Diego Tyszka Martinez ${ }^{2}$ (D) 0000-0002-7275-8043 \\ Sidney Fernando Caldeira ${ }^{1}$ (1) 0000-0001-6042-4313 \\ Rodrigo Adversi Silva ${ }^{1}$ (1) 0000-0002-5849-0386 \\ Felipe Andrade Antonieto ${ }^{3}$ (1) 0000-0001-8844-5140
}

\begin{abstract}
Urban trees/vegetation are one of the factors related to a better quality of life of the population. Therefore, knowledge on the structure and quality of urban green areas is important to implement actions that intensify this benefit. This study aimed to quantify the floristic composition of the Universidade Federal do Mato Grosso, in Cuiabá. A census was conducted to count individuals with soil circumference $\geq 15.7 \mathrm{~cm}$, wherein 190 species were identified, with Fabaceae being the most representative family at $24.2 \%$. Of the total, $23.7 \%$ are exotic species to Brazil and $62.6 \%$ are native to the Cerrado. The most abundant species were Licania tomentosa, with $8.9 \%$ of individuals, Mangifera indica at $7.8 \%$, Jacaranda cuspidifolia with $6.3 \%$, Handroanthus impetiginosus with $5.4 \%$, and Anadenanthera peregrine with $4.4 \%$. The university campus houses a high floristic richness and diversity of species, with an adequate proportion of Brazilian native species. However, its composition needs to be adapted to comply with municipal legislation.
\end{abstract}

Keywords: afforestation, diversity, public spaces.

\section{INTRODUCTION AND OBJECTIVES}

Human occupation in cities most often increases fast, in disorder and without planning, with direct consequences on quality of life (Pinheiro \& Souza, 2017). In this way, performing and deepening studies related to urban afforestation is necessary, due to the contribution of urban greenery to an improved environmental quality; a fact that also generates positive psychological and physical effects for its inhabitants (McHale et al., 2007).

Green areas are the results of landscaping processes in cities which have several influence of man, and which divide cultural green areas such as plazas, squares and gardens, and urban forest fragments, which are basically anthropized forest remnants due to the urbanization process (Biondi, 2015).

The ecological function of these areas is related to the quality of the urban environment, favoring climate mitigation since the natural elements that compose these spaces attenuate the impacts resulting from industrialization. There is a feeling of well-being in these places in different environments, which enables community permanence and also provides areas for meetings and social relationships (Loboda \& Angelis, 2005).

Green areas structure ecological corridors, especially with the presence of native fruit species, which helps developing a richer fauna and is a very important element for maintaining the biodiversity of urban ecosystems and indicators of environmental quality in cities (Alencar \& Cardoso, 2015).

Knowledge of the floristic composition of urban green areas is fundamental for environmental balance and should be part of frequent study programs in order to value the landscape and the local physiognomy with implantation of species adapted to each situation. In addition to the benefits of vegetation to human health, the ecological peculiarity of the region should also be considered in order to preserve the genetic identity (Kramer \& Krupek, 2012).

\footnotetext{
${ }^{1}$ Universidade Federal de Mato Grosso (UFMT), Cuiabá, MT, Brasil

${ }^{2}$ Universidade Federal do Paraná (UFPR), Curitiba, PR, Brasil

${ }^{3}$ Instituto Nacional de Pesquisas da Amazônia (INPA), Manaus, AM, Brasil
} 
For urban afforestation to perform its intended function, it is necessary to prior plan planted green areas, since many of the benefits that the population receives from ecosystems in order to guarantee their well-being depends on tree species diversification (Morgenroth et al., 2016). In this sense, Brazilian academic institutions, with their potential for scientific production, should develop floristic projects that consider the peculiar characteristics of the species and use them for implanting forestry with superior quality (Eisenlohr et al., 2008).

The Universidade Federal do Mato Grosso (UFMT) campus is characterized as an important green area fragment, which contributes to the quality of life of local inhabitants and provides environmental services to the city. With an apparent tree flora diversity comprising native and exotic species, it is located in an original Cerrado region, with the most part being artificial trees implanted in its construction phase.

Knowledge of the existing tree species on the campus is important for planning urban afforestation in order to better use the characteristics of each individual in the environment, as well as to know its richness and diversity. This fact supports new native species plantations, and the removal of exotic species if necessary.

In view of the above, this study aimed to learn about the floristic composition of UFMT afforestation at the Cuiabá campus to show the specific aspects that can be implemented to improve its structure.

\section{MATERIALS AND METHODS}

This study was conducted at the UFMT, Cuiabá campus, circumscribed to the coordinates $15^{\circ} 35^{\prime} 46^{\prime \prime}$ South latitude and $56^{\circ} 5^{\prime} 48^{\prime \prime}$ West longitude. The campus extends to an area of about 76 ha in the Boa Esperança neighborhood of Cuiabá, Mato Grosso.

Most of the vegetation that makes up UFMT was planted in the early 1970s, with an apparent tree species diversity native to the Cerrado. Later plantings were carried out over the years by student and commemorative activities or to obtain shading in order to mitigate the effect of heat.

The climate of the region is Aw (Köppen-Geiger) with well-defined dry winter and rainy summer seasons, and average annual temperature of $25^{\circ} \mathrm{C}$. The average local altitude is $150 \mathrm{~m}$, with average annual rainfall ranging between 1,300 mm.year ${ }^{-1}$ and $1600 \mathrm{~mm}$.year ${ }^{-1}$ (Alvares et al., 2013).

According to the Embrapa soil classification (Santos et al., 2013), the predominant soil of the region is Dystrophic Redyellow Argisol. The local vegetation comprises a large diversity of native and exotic tree species, with emphasis on Cerrado species, a predominant forest typology in the capital of Mato Grosso. The city of Cuiabá has an approximate population of 590,000 inhabitants in an area of 3,293,537 $\mathrm{km}^{2}$ (IBGE, 2017).

Data were collected via a census of tree vegetation at UFMT, Cuiabá campus, conducted from July 2016 to January 2017. Data were recorded in pre-structured tables containing tree number and species information. Using a measuring tape, individuals with a circumference at $1.30 \mathrm{~m}$ above ground level (breast-height) (CBH $1.3 \mathrm{~m}$ ) equal to or greater than $15.7 \mathrm{~cm}$ were included, which is equivalent to 5 tape $\mathrm{cm}$ in diameter at $1.3 \mathrm{~m}(\mathrm{DBH} 1.3 \mathrm{~m})$. Each tree received a metal plate with an identification number, which enabled indexing information on the family, genus, and species when possible.

The known species were identified on site, while the unknown species had their botanical material collected for later identification at the Laboratory of Dendrology, School of Forestry Engineering, UFMT.

The species were organized according to families registered in the Angiosperm Phylogeny Group III (Stevens, 2016), and scientific names were checked on the Missouri Botanical Garden (2016) portal. The species were also classified according to their origin as native or exotic to Brazil, as to their natural occurrence in the phytogeographical domain of the Cerrado to which the municipality of Cuiabá belongs, and as to leaf deciduousness, followed by the reference of the Brazil 2020 Flora program currently under construction (Coppetec, c2019).

After data collection, the species were divided into 4 groups according to their relevant characteristic for use in urban afforestation. The created groups were: Palm trees, which gathered the species of the Arecaceae family; Fruit trees, which grouped species that produce fleshy fruits; Ornamental, with species that show intense and striking flowering; and Others, which gathered the species that did not fit into the previous groups, but provided other environmental regulation services to the city, such as shade, climatic mitigation, air pollution retention, noise retention, atmospheric carbon retention and rainwater interception.

Data were analyzed by descriptive statistics starting from structuring the table, with the calculations of relative abundance by species (Equation 1) being performed through the MS Excel 2016:

$$
\mathrm{Ab} \cdot \mathrm{R}=\mathrm{Ab} \cdot \mathrm{A}^{\star} 100 / \mathrm{N}
$$

Ab.R: number of individuals of a given species; Ab.A: absolute abundance; $\mathrm{N}$ : total number of individuals. 
Diversity was determined using the Shannon-Weiner Indexes (Equation 2) and Simpson Index (Equation 3) shown by Odum (2012), calculated by the R program (R Development Core Team, 2015):

$$
\mathrm{H}^{\prime}=-\sum\left((\mathrm{ni} / \mathrm{N})^{\star} \ln (\mathrm{ni} / \mathrm{N})\right)
$$

$H^{\prime}$ : Shannon Index; ni: number of individuals in the $i^{\text {th }}$ species; $\mathrm{N}$ : total number of individuals.

$$
\mathrm{D}=\sum\left(\mathrm{ni}^{\star}(\mathrm{ni}-1) /(\mathrm{N}(\mathrm{N}-1))\right.
$$

D: Simpson Index; ni: number of individuals in the $\mathrm{i}^{\text {th }}$ species; $\mathrm{N}$ : total number of individuals.

\section{RESULTS AND DISCUSSION}

We surveyed 4817 shrub and tree individuals, of which two were not classified, while 14 were classified regarding family, 66 were classified in genus and 80 were dead. The richness was 189 forest species, with 182 trees and 7 palm trees, distributed across 136 genera and 48 botanical families.

The most representative family of species was Fabaceae, with $24.2 \%$, followed by Malvaceae (7.9\%), Myrtaceae (6.8\%), Bignoniaceae (5.8\%), and others with values less than $4 \%$. The ten most representative families characterized $63.2 \%$ of the total trees. Among the species, Licania tomentosa was the most abundant with $8.9 \%$, followed by Mangifera indica (7.8\%), Jacaranda cuspidifolia (6.3\%), Handroanthus impetiginosus (5.4\%), and Anadenanthera peregrina (4.4\%) (Table 1).

Table 1. Floristic composition, origin (Or.), deciduity (Dec.) groups and relative abundance (RAb.) of species in the afforestation of

\begin{tabular}{|c|c|c|c|c|c|c|c|c|}
\hline \multirow{2}{*}{$\mathbf{N}$} & \multirow{2}{*}{ Species, genus, family or absence of classification } & \multirow{2}{*}{ Or. } & \multirow{2}{*}{ Dec. } & \multicolumn{4}{|c|}{ Groups } & \multirow{2}{*}{ RAb. } \\
\hline & & & & $\mathbf{P}$ & $\mathbf{F}$ & $\mathbf{0}$ & Oth. & \\
\hline 1 & Licania tomentosa (Benth.) Fritsch & $\mathrm{N}$ & $\mathrm{PR}$ & & & & $\mathrm{x}$ & 8.9 \\
\hline 2 & Mangifera indica $\mathrm{L}$. & $\mathrm{E}$ & PR & & $\mathrm{x}$ & & & 7.8 \\
\hline 3 & Jacaranda cuspidifolia Mart. & $\mathrm{N}^{*}$ & $\mathrm{D}$ & & & $\mathrm{x}$ & & 6.3 \\
\hline 4 & Handroanthus impetiginosus (Mart. ex DC.) Mattos & $\mathrm{N}^{*}$ & $\mathrm{D}$ & & & & $\mathrm{x}$ & 5.4 \\
\hline 5 & Anadenanthera peregrina (L.) Speg. & $\mathrm{N}^{*}$ & $\mathrm{D}$ & & & & $\mathrm{x}$ & 4.4 \\
\hline 6 & Cariniana rubra Gardner ex Miers & $\mathrm{N}^{*}$ & $\mathrm{D}$ & & & & $\mathrm{x}$ & 3.5 \\
\hline 7 & Genipa americana L. & $\mathrm{N}^{*}$ & $\mathrm{D}$ & & $\mathrm{x}$ & & & 3.5 \\
\hline 8 & Astronium fraxinifolium Schott & $\mathrm{N}^{*}$ & $\mathrm{D}$ & & & & $\mathrm{x}$ & 3.1 \\
\hline 9 & Acrocomia aculeata (Jacq.) Lodd. ex Mart. & $\mathrm{N}^{*}$ & $\mathrm{PR}$ & $\mathrm{x}$ & & & & 3 \\
\hline 10 & Dipteryx alata Vogel & $\mathrm{N}^{*}$ & $\mathrm{PR}$ & & $\mathrm{x}$ & & & 2.8 \\
\hline 11 & Swietenia macrophylla King & $\mathrm{N}^{*}$ & $\mathrm{D}$ & & & & $\mathrm{x}$ & 2.2 \\
\hline 12 & Cordia glabrata (Mart.) A. DC. & $\mathrm{N}^{*}$ & $\mathrm{D}$ & & & $\mathrm{x}$ & & 2.1 \\
\hline 13 & Psidium guajava L. & $\mathrm{E}$ & $\mathrm{PR}$ & & $\mathrm{x}$ & & & 1.6 \\
\hline 14 & Anacardium occidentale $\mathrm{L}$. & $\mathrm{N}^{*}$ & $\mathrm{D}$ & & $\mathrm{x}$ & & & 1.5 \\
\hline 15 & Hevea brasiliensis (Willd. ex A. Juss.) Müll. Arg. & $\mathrm{N}$ & $\mathrm{D}$ & & & & $\mathrm{x}$ & 1.5 \\
\hline 16 & Cecropia pachystachya Trec. & $\mathrm{N}^{*}$ & $\mathrm{D}$ & & & & $\mathrm{x}$ & 1.4 \\
\hline 17 & Curatella americana $\mathrm{L}$. & $\mathrm{N}^{*}$ & $\mathrm{D}$ & & & & $\mathrm{x}$ & 1.3 \\
\hline 18 & Albizia lebbeck (L.) Benth. & $\mathrm{E}$ & $\mathrm{D}$ & & & & $\mathrm{x}$ & 1.2 \\
\hline 19 & Samanea tubulosa (Benth.) Barneby \& J.W. Grimes & $\mathrm{N}^{*}$ & $\mathrm{D}$ & & $\mathrm{x}$ & & & 1.2 \\
\hline 20 & Handroanthus avellanedae (Lorentz ex Griseb.) Mattos & $\mathrm{N}$ & $\mathrm{D}$ & & & $\mathrm{x}$ & & 1.2 \\
\hline 21 & Delonix regia (Bojer ex Hook.) Raf. & $\mathrm{E}$ & $\mathrm{D}$ & & & $\mathrm{x}$ & & 1.1 \\
\hline 22 & Myracrodruon urundeuva Allemão & $\mathrm{N}^{*}$ & $\mathrm{D}$ & & & & $\mathrm{x}$ & 1.1 \\
\hline 23 & Inga laurina (Sw.) Willd. & $\mathrm{N}^{*}$ & $\mathrm{PR}$ & & & & $\mathrm{x}$ & 1.1 \\
\hline 24 & Handroanthus aureus Mattos & $\mathrm{N}^{*}$ & $\mathrm{D}$ & & & $\mathrm{x}$ & & 1.0 \\
\hline 25 & Enterolobium contortisiliquum (Vell.) Morong & $\mathrm{N}^{*}$ & $\mathrm{D}$ & & & & $\mathrm{x}$ & 1.0 \\
\hline 26 & Guazuma ulmifolia Pers. & $\mathrm{N}^{*}$ & $\mathrm{D}$ & & $\mathrm{x}$ & & & 1.0 \\
\hline 27 & Vochysia divergens Pohl & $\mathrm{N}^{*}$ & $\mathrm{PR}$ & & & & $\mathrm{x}$ & 0.9 \\
\hline
\end{tabular}
UFMT campus Cuiabá, 2017. 
Table 1. Continued..

\begin{tabular}{|c|c|c|c|c|c|c|c|c|}
\hline \multirow{2}{*}{$\mathbf{N}$} & \multirow{2}{*}{ Species, genus, family or absence of classification } & \multirow{2}{*}{ Or. } & \multirow{2}{*}{ Dec. } & \multicolumn{4}{|c|}{ Groups } & \multirow[t]{2}{*}{ RAb. } \\
\hline & & & & $\mathbf{P}$ & $\mathbf{F}$ & $\mathbf{O}$ & Oth. & \\
\hline 28 & Mimosa caesalpiniifolia Benth. & $\mathrm{N}^{*}$ & $\mathrm{D}$ & & & & $\mathrm{x}$ & 0.9 \\
\hline 29 & Cassia siamea Lam. & $\mathrm{N}^{*}$ & PR & & & $\mathrm{x}$ & & 0.8 \\
\hline 30 & Gmelina arborea Roxb. ex Sm. & $\mathrm{E}$ & $\mathrm{D}$ & & $\mathrm{x}$ & & & 0.7 \\
\hline 31 & Anadenanthera falcata (Benth.) Speg. & $\mathrm{N}^{*}$ & $\mathrm{D}$ & & & & $\mathrm{x}$ & 0.7 \\
\hline 32 & Handroanthus serratifolius (Vahl) S.O. Grose & $\mathrm{N}^{*}$ & $\mathrm{D}$ & & & $\mathrm{x}$ & & 0.7 \\
\hline 33 & Leucaena leucocephala (Lam.) de Wit & $\mathrm{E}$ & $\mathrm{D}$ & & & & $\mathrm{x}$ & 0.6 \\
\hline 34 & Cordia aff. myxa L. & $\mathrm{E}$ & $\mathrm{PR}$ & & & & $\mathrm{x}$ & 0.6 \\
\hline 35 & Cocos nucifera L. & $\mathrm{N}$ & PR & $\mathrm{x}$ & & & & 0.6 \\
\hline 36 & Caryocar brasiliense A. St.-Hil. & $\mathrm{N}^{*}$ & $\mathrm{D}$ & & $\mathrm{x}$ & & & 0.6 \\
\hline 38 & Buchenavia tomentosa Eichler & $\mathrm{N}^{*}$ & $\mathrm{D}$ & & $\mathrm{x}$ & & & 0.5 \\
\hline 39 & Casearia sylvestris Sw. & $\mathrm{N}^{*}$ & $\mathrm{D}$ & & $\mathrm{x}$ & & & 0.5 \\
\hline 40 & Magonia pubescens A. St.-Hil. & $\mathrm{N}^{*}$ & $\mathrm{D}$ & & & & $\mathrm{x}$ & 0.5 \\
\hline 41 & Pithecellobium hassleri Chodat & $\mathrm{N}$ & $\mathrm{D}$ & & & & $\mathrm{x}$ & 0.5 \\
\hline 42 & Vatairea macrocarpa (Benth.) Ducke & $\mathrm{N}^{*}$ & $\mathrm{D}$ & & & $\mathrm{x}$ & & 0.5 \\
\hline 43 & Tamarindus indica $\mathrm{L}$. & $\mathrm{E}$ & $\mathrm{D}$ & & $\mathrm{x}$ & & & 0.5 \\
\hline 44 & Bowdichia virgilioides Kunth & $\mathrm{N}^{*}$ & $\mathrm{D}$ & & & $\mathrm{x}$ & & 0.4 \\
\hline 45 & Amburana acreana (Ducke) A.C. Sm. & $\mathrm{N}$ & $\mathrm{D}$ & & & & $\mathrm{x}$ & 0.4 \\
\hline 46 & Ceiba boliviana Britten \& Baker $\mathrm{f}$. & $\mathrm{N}^{*}$ & $\mathrm{D}$ & & & $\mathrm{x}$ & & 0.4 \\
\hline 47 & Talisia esculenta (A. St.-Hil.) Radlk. & $\mathrm{N}^{*}$ & $\mathrm{D}$ & & $\mathrm{x}$ & & & 0.4 \\
\hline 50 & Hymenaea courbaril L. & $\mathrm{N}^{*}$ & $\mathrm{D}$ & & $\mathrm{x}$ & & & 0.3 \\
\hline 51 & Luehea paniculata Mart. & $\mathrm{N}^{*}$ & $\mathrm{D}$ & & & & $\mathrm{x}$ & 0.3 \\
\hline 52 & Qualea grandiflora Mart. & $\mathrm{N}^{*}$ & $\mathrm{D}$ & & & & $\mathrm{x}$ & 0.3 \\
\hline 53 & Terminalia catappa L. & $\mathrm{E}$ & $\mathrm{D}$ & & & & $\mathrm{x}$ & 0.3 \\
\hline 54 & Ficus benjamina L. & $\mathrm{N}^{*}$ & PR & & $\mathrm{x}$ & & & 0.3 \\
\hline 55 & Inga sp. & $\mathrm{N}^{*}$ & PR & & & & $\mathrm{x}$ & 0.3 \\
\hline 56 & Protium heptaphyllum (Aubl.) Marchand & $\mathrm{N}^{*}$ & PR & & $\mathrm{x}$ & & & 0.3 \\
\hline 57 & Cassia fistula L. & $\mathrm{E}$ & $\mathrm{D}$ & & & $\mathrm{x}$ & & 0.3 \\
\hline 58 & Hymenaea stigonocarpa Mart. ex Hayne & $\mathrm{N}^{*}$ & $\mathrm{D}$ & & $\mathrm{x}$ & & & 0.3 \\
\hline 59 & Vochysia haenkeana Mart. & $\mathrm{N}^{*}$ & $\mathrm{D}$ & & & & $\mathrm{x}$ & 0.3 \\
\hline 60 & Aspidosperma riedelii Müll.Arg & $\mathrm{N}$ & $\mathrm{D}$ & & & & $\mathrm{x}$ & 0.3 \\
\hline 61 & Erythroxylum suberosum A. St.-Hil. & $\mathrm{N}^{*}$ & $\mathrm{D}$ & & $\mathrm{x}$ & & & 0.3 \\
\hline 62 & Eucalyptussp. 2 & $\mathrm{E}$ & $\mathrm{D}$ & & & & $\mathrm{x}$ & 0.3 \\
\hline 63 & Luehea grandiflora Mart. & $\mathrm{N}^{*}$ & $\mathrm{D}$ & & & & $\mathrm{x}$ & 0.3 \\
\hline 64 & Murraya paniculata (L.) Jack & $\mathrm{E}$ & PR & & $\mathrm{x}$ & & & 0.3 \\
\hline 65 & Peltophorum dubium (Spreng.) Taub. & $\mathrm{N}^{*}$ & $\mathrm{D}$ & & & $\mathrm{x}$ & & 0.3 \\
\hline 66 & Spondias purpurea L. & $\mathrm{E}$ & $\mathrm{D}$ & & $\mathrm{x}$ & & & 0.3 \\
\hline 67 & Cedrela fissilis Vell. & $\mathrm{N}^{*}$ & $\mathrm{D}$ & & & & $\mathrm{x}$ & 0.2 \\
\hline 68 & Chorisia speciosa A. St.-Hil. & $\mathrm{N}^{*}$ & $\mathrm{D}$ & & & $\mathrm{x}$ & & 0.2 \\
\hline 69 & Cordia trichotoma (Vell.) Arráb. ex Steud. & $\mathrm{N}^{*}$ & $\mathrm{D}$ & & & $x$ & & 0.2 \\
\hline
\end{tabular}


Table 1. Continued...

\begin{tabular}{|c|c|c|c|c|c|c|c|c|}
\hline \multirow{2}{*}{$\mathbf{N}$} & \multirow{2}{*}{ Species, genus, family or absence of classification } & \multirow{2}{*}{ Or. } & \multirow{2}{*}{ Dec. } & \multicolumn{4}{|c|}{ Groups } & \multirow[t]{2}{*}{ RAb. } \\
\hline & & & & $\mathbf{P}$ & $\mathbf{F}$ & $\mathbf{O}$ & Oth. & \\
\hline 71 & Handroanthus roseo-albus (Ridl.) Mattos & $\mathrm{N}^{*}$ & $\mathrm{D}$ & & & $\mathrm{x}$ & & 0.2 \\
\hline 72 & Physocalymma scaberrimum Pohl & $\mathrm{N}^{*}$ & $\mathrm{D}$ & & & $\mathrm{x}$ & & 0.2 \\
\hline 73 & Platypodium elegans Vogel & $\mathrm{N}^{*}$ & $\mathrm{D}$ & & & $\mathrm{x}$ & & 0.2 \\
\hline 74 & Andira cuyabensis Benth. & $\mathrm{N}^{*}$ & $\mathrm{D}$ & & & & $\mathrm{x}$ & 0.2 \\
\hline 75 & Clitoria fairchildiana R.A. Howard & $\mathrm{N}^{*}$ & $\mathrm{D}$ & & & & $\mathrm{x}$ & 0.2 \\
\hline 76 & Copaifera langsdorffii Desf. & $\mathrm{N}^{*}$ & $\mathrm{D}$ & & & & $\mathrm{x}$ & 0.2 \\
\hline 77 & Malpighia glabra L. & $\mathrm{E}$ & PR & & $\mathrm{x}$ & & & 0.2 \\
\hline 78 & Muntingia calabura L. & $\mathrm{N}$ & $\mathrm{D}$ & & $\mathrm{x}$ & & & 0.2 \\
\hline 79 & Pachira aquatica Aubl. & $\mathrm{N}$ & $\mathrm{D}$ & & $\mathrm{x}$ & & & 0.2 \\
\hline 80 & Terminalia argentea Mart. ex Succ. & $\mathrm{N}^{*}$ & $\mathrm{D}$ & & & & $\mathrm{x}$ & 0.2 \\
\hline 81 & Guettarda viburnoides Cham. \& Schltdl. & $\mathrm{N}^{*}$ & $\mathrm{D}$ & & $\mathrm{x}$ & & & 0.2 \\
\hline 82 & Handroanthus chrysotrichus (Mart. ex A. DC.) Mattos & $\mathrm{N}^{*}$ & $\mathrm{D}$ & & & $\mathrm{x}$ & & 0.2 \\
\hline 83 & Orbignya speciosa (Mart. ex Spreng.) Barb. Rodr. & $\mathrm{N}^{*}$ & $\mathrm{PR}$ & $\mathrm{x}$ & & & & 0.2 \\
\hline 84 & Platymiscium pinnatum (Jacq.) Dugand & $\mathrm{N}$ & $\mathrm{D}$ & & & $\mathrm{x}$ & & 0.2 \\
\hline 85 & Tectona grandis L. f. & $\mathrm{E}$ & $\mathrm{D}$ & & & & $\mathrm{x}$ & 0.2 \\
\hline 86 & Alibertia edulis (Rich.) A. Rich. ex DC. & $\mathrm{N}^{*}$ & PR & & $\mathrm{x}$ & & & 0.2 \\
\hline 87 & Guazuma tomentosa Kunth & $\mathrm{N}^{*}$ & PR & & $\mathrm{x}$ & & & 0.2 \\
\hline 88 & Tapirira guianensis Aubl. & $\mathrm{N}^{*}$ & PR & & $\mathrm{x}$ & & & 0.2 \\
\hline 89 & Vitex cymosa Bertero ex Spreng. & $\mathrm{N}^{*}$ & $\mathrm{D}$ & & $\mathrm{x}$ & & & 0.2 \\
\hline 90 & Ficus gomelleira Kunth \& C.D. Bouché & $\mathrm{E}$ & $\mathrm{D}$ & & $\mathrm{x}$ & & & 0.2 \\
\hline 91 & Machaerium aculeatum Raddi & $\mathrm{N}^{*}$ & $\mathrm{D}$ & & & & $\mathrm{x}$ & 0.2 \\
\hline 92 & Rubiaceae sp. & - & - & & & & $\mathrm{x}$ & 0.2 \\
\hline 93 & Sterculia striata A. St.-Hil. \& Naudin & $\mathrm{N}^{*}$ & $\mathrm{D}$ & & & & $\mathrm{x}$ & 0.2 \\
\hline 94 & Ficus insipida Willd. & $\mathrm{N}$ & $\mathrm{D}$ & & $\mathrm{x}$ & & & 0.1 \\
\hline 95 & Pseudobombax longiflorum (Mart.) A. Robyns & $\mathrm{N}^{*}$ & $\mathrm{D}$ & & & & $\mathrm{x}$ & 0.1 \\
\hline 96 & Spondias dulcis Parkinson & $\mathrm{E}$ & $\mathrm{D}$ & & $\mathrm{x}$ & & & 0.1 \\
\hline 97 & Syzygium jambolanum (Lam.) DC. & $\mathrm{E}$ & PR & & $\mathrm{x}$ & & & 0.1 \\
\hline 98 & Acosmium subelegans (Mohlenbr.) Yakovlev & $\mathrm{N}^{*}$ & $\mathrm{D}$ & & & & $\mathrm{x}$ & 0.1 \\
\hline 99 & Annona crassiflora Mart. & $\mathrm{N}^{*}$ & $\mathrm{D}$ & & $\mathrm{x}$ & & & 0.1 \\
\hline 100 & Aspidosperma subincanum Mart. ex A. DC. & $\mathrm{N}^{*}$ & $\mathrm{D}$ & & & & $\mathrm{x}$ & 0.1 \\
\hline 101 & Bauhinia variegata $\mathrm{L}$. & $\mathrm{N}^{*}$ & $\mathrm{D}$ & & & $\mathrm{x}$ & & 0.1 \\
\hline 102 & Citrus sp. 3 & $\mathrm{E}$ & PR & & $\mathrm{x}$ & & & 0.1 \\
\hline 103 & Hirtella gracilipes (Hook. f.) Prance & $\mathrm{N}^{*}$ & $\mathrm{D}$ & & $\mathrm{x}$ & & & 0.1 \\
\hline 104 & Machaerium acutifolium Vogel & $\mathrm{N}^{*}$ & PR & & & & $\mathrm{x}$ & 0.1 \\
\hline 105 & Pouteria gardneri (Mart. \& Miq.) Baehni & $\mathrm{N}^{*}$ & $\mathrm{D}$ & & $\mathrm{x}$ & & & 0.1 \\
\hline 106 & Zanthoxylum rhoifolium Lam. & $\mathrm{N}^{*}$ & PR & & $\mathrm{x}$ & & & 0.1 \\
\hline 107 & Apeiba tibourbou Aubl. & $\mathrm{N}^{*}$ & PR & & & & $\mathrm{x}$ & 0.1 \\
\hline 108 & Aspidosperma cylindrocarpon Muell. Arg. & $\mathrm{N}^{*}$ & PR & & & & $\mathrm{x}$ & 0.1 \\
\hline 109 & Brosimum gaudichaudii Trécul & $\mathrm{N}^{*}$ & $\mathrm{D}$ & & $\mathrm{x}$ & & & 0.1 \\
\hline 110 & Cordia ecalyculata Vell. & $\mathrm{N}$ & $\mathrm{D}$ & & $\mathrm{x}$ & & & 0.1 \\
\hline 111 & Cybistax antisyphilitica (Mart.) Mart. & $\mathrm{N}^{*}$ & $\mathrm{D}$ & & & $\mathrm{x}$ & & 0.1 \\
\hline 112 & Eriotheca gracilipes (K. Schum.) A. Robyns & $\mathrm{N}^{*}$ & $\mathrm{D}$ & & & & $\mathrm{x}$ & 0.1 \\
\hline 113 & Inga edulis Mart. & $\mathrm{N}^{*}$ & PR & & & & $\mathrm{x}$ & 0.1 \\
\hline
\end{tabular}


Table 1. Continued...

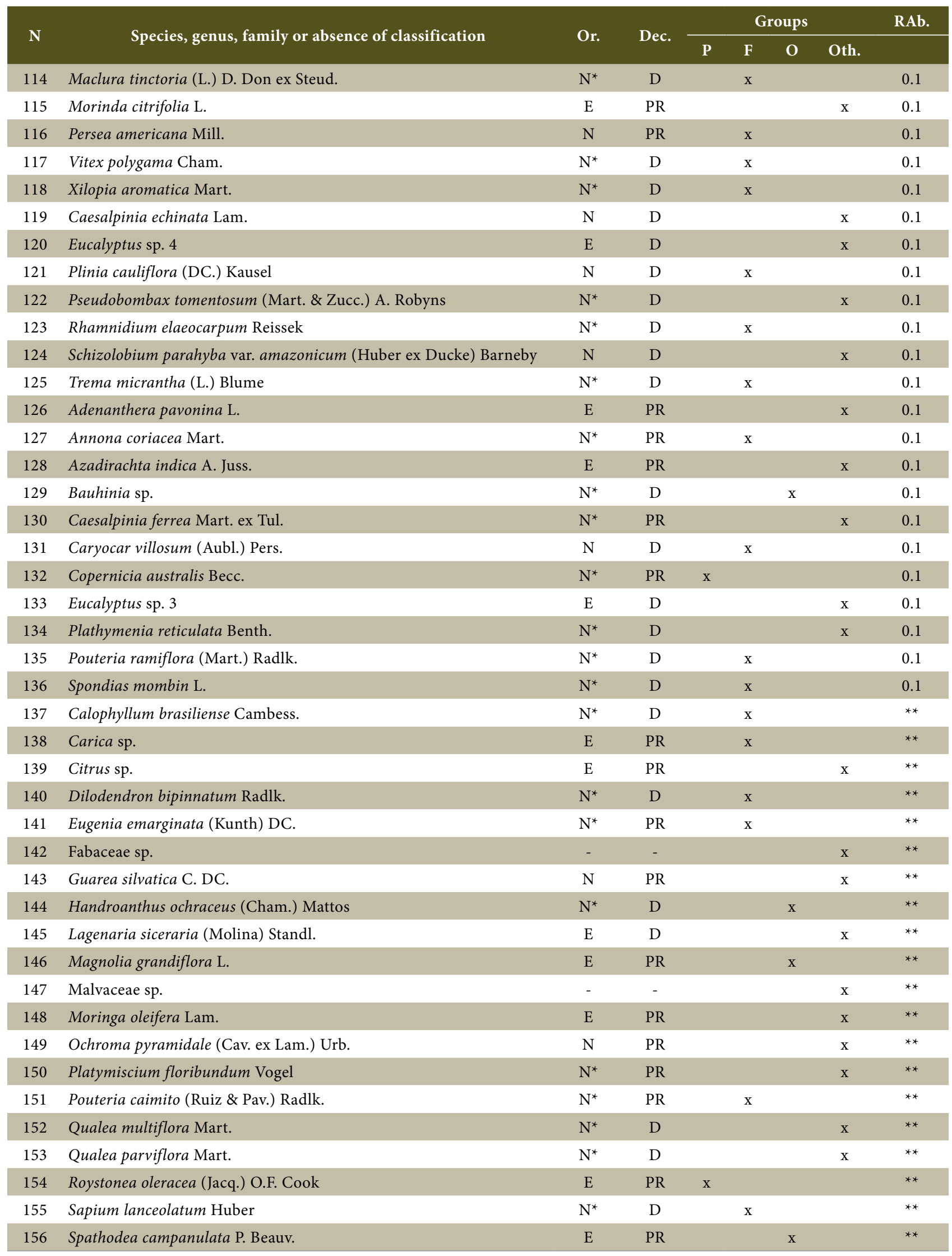


Table 1. Continued...

\begin{tabular}{|c|c|c|c|c|c|c|c|c|}
\hline \multirow{2}{*}{$\mathbf{N}$} & \multirow{2}{*}{ Species, genus, family or absence of classification } & \multirow{2}{*}{ Or. } & \multirow{2}{*}{ Dec. } & \multicolumn{4}{|c|}{ Groups } & \multirow[t]{2}{*}{ RAb. } \\
\hline & & & & $\mathbf{P}$ & $\mathbf{F}$ & $\mathbf{O}$ & Oth. & \\
\hline 157 & Syzygium cumini (L.) Skeels & $\mathrm{E}$ & $\mathrm{D}$ & & $\mathrm{x}$ & & & $* *$ \\
\hline 158 & Triplaris americana $\mathrm{L}$. & $\mathrm{N}^{*}$ & PR & & & $\mathrm{x}$ & & $* *$ \\
\hline 159 & Annona reticulata $\mathrm{L}$. & $\mathrm{E}$ & PR & & $\mathrm{x}$ & & & $* *$ \\
\hline 160 & Attalea phalerata Mart. ex Spreng. & $\mathrm{N}^{*}$ & $\mathrm{PR}$ & $\mathrm{x}$ & & & & ** \\
\hline 161 & Averrhoa carambola $\mathrm{L}$. & $\mathrm{E}$ & PR & & $\mathrm{x}$ & & & $* *$ \\
\hline 162 & Bauhinia cuiabensis Steud. & $\mathrm{E}^{*}$ & $\mathrm{D}$ & & & $\mathrm{x}$ & & ** \\
\hline 163 & Bauhinia rufa (Bong.) Steud. & $\mathrm{E}^{*}$ & $\mathrm{D}$ & & & $\mathrm{x}$ & & $* *$ \\
\hline 164 & Bauhinia sp. 2 & $E^{*}$ & $\mathrm{D}$ & & & $\mathrm{x}$ & & ** \\
\hline 165 & Bauhinia sp. 3 & $\mathrm{E}^{*}$ & $\mathrm{D}$ & & & $\mathrm{x}$ & & $* *$ \\
\hline 166 & Bertholletia excelsa Bonpl. & $\mathrm{N}$ & $\mathrm{D}$ & & $\mathrm{x}$ & & & ** \\
\hline 167 & Bixa orellana $\mathrm{L}$. & $\mathrm{N}^{*}$ & PR & & & & $\mathrm{x}$ & ** \\
\hline 168 & Byrsonima coccolobifolia Kunth & $\mathrm{N}^{*}$ & $\mathrm{D}$ & & $\mathrm{x}$ & & & ** \\
\hline 169 & Capparis retusa Griseb. & $\mathrm{E}$ & $\mathrm{PR}$ & & $\mathrm{x}$ & & & ** \\
\hline 170 & Citrus sp. 2 & $\mathrm{E}$ & PR & & & & $\mathrm{x}$ & ** \\
\hline 171 & Coccoloba mollis Casar. & $\mathrm{N}^{*}$ & $\mathrm{D}$ & & $\mathrm{x}$ & & & $* *$ \\
\hline 172 & Copernicia prunifera (Mill.) H.E. Moore & $\mathrm{N}^{*}$ & PR & $\mathrm{x}$ & & & & ** \\
\hline 173 & Dracaena sp. & $\mathrm{E}$ & $\mathrm{PR}$ & & & & $\mathrm{x}$ & ** \\
\hline 174 & Erythrina mulungu Mart. ex Benth. & $\mathrm{E}$ & $\mathrm{D}$ & & & $\mathrm{x}$ & & ** \\
\hline 175 & Eugenia bimarginata DC. & $\mathrm{N}^{*}$ & PR & & $\mathrm{x}$ & & & $* *$ \\
\hline 176 & Euphorbiaceae sp. & - & - & & & & $\mathrm{x}$ & ** \\
\hline 177 & Garcinia gardneriana (Planch. \& Triana) Zappi & $\mathrm{N}^{*}$ & $\mathrm{PR}$ & & $\mathrm{x}$ & & & ** \\
\hline 178 & Indeterminda 1 & - & - & & & & $\mathrm{x}$ & ** \\
\hline 179 & Indeterminda 2 & - & - & & & & $\mathrm{x}$ & ** \\
\hline 180 & Jatropha curcas L. & $\mathrm{E}^{*}$ & $\mathrm{D}$ & & & & $\mathrm{x}$ & ** \\
\hline 181 & Khaya ivorensis A. Chev. & $\mathrm{E}$ & $\mathrm{PR}$ & & & & $\mathrm{x}$ & $* *$ \\
\hline 182 & Malvaceae sp. 2 & - & - & & & & $\mathrm{x}$ & ** \\
\hline 183 & Pinus caribaea Morelet & $\mathrm{E}$ & $\mathrm{PR}$ & & & & $\mathrm{x}$ & ** \\
\hline 184 & Plumeria rubra L. & $\mathrm{E}$ & $\mathrm{D}$ & & & $\mathrm{x}$ & & ** \\
\hline 185 & Psidium cattleyanum Sabine & $\mathrm{N}^{*}$ & $\mathrm{PR}$ & & $\mathrm{x}$ & & & ** \\
\hline 186 & Psidium sp. & $\mathrm{E}$ & $\mathrm{D}$ & & $\mathrm{x}$ & & & ** \\
\hline 187 & Roupala montana Aubl. & $\mathrm{N}^{*}$ & $\mathrm{D}$ & & & & $\mathrm{x}$ & ** \\
\hline 188 & Schefflera morototoni (Aubl.) Maguire. Steyerm. \& Frodin & $\mathrm{N}^{*}$ & PR & & $\mathrm{x}$ & & & ** \\
\hline 189 & Stryphnodendron adstringens (Mart.) Coville & $\mathrm{N}^{*}$ & $\mathrm{D}$ & & & & $\mathrm{x}$ & $* *$ \\
\hline 190 & Zygia sp. & $\mathrm{N}^{*}$ & $\mathrm{D}$ & & & & $\mathrm{x}$ & ** \\
\hline Total & & - & - & 7 & 71 & 32 & 80 & 100 \\
\hline
\end{tabular}

Origin (Or.): Native to Brazil (N), Exotic (E). Natural occurrence in the phytogeographical domain of the Cerrado $\left(^{*}\right)$; Deciduity (Dec.): Deciduous (D), Perennial (PR); Groups: Palm trees (P), Fruitful (F), Ornamental (O), Other (Oth.); Value $<0.1{ }^{(*)}$.

The values of the twelve most frequent species represent $53.2 \%$ of all individuals found on campus. Milano \& Dalcin (2000) consider that the quantity of individuals of a species should not exceed $15 \%$ of the total population. In this case, the species from the UFMT fall within the recommendations of these authors.
Regarding deciduity, $64.7 \%$ of the species were deciduous, $31.6 \%$ perennial and $3.7 \%$ were not classified due to lack of identification. When compared to the proportion of autochthonous species on campus, this result can be explained since the majority of naturally occurring Cerrado species are characterized by leaf loss in the dry season. However, 
Cuiabá is a city with high temperatures throughout the year, especially during August to October, so that it is necessary to implant perennial species such as Licania tomentosa, the most abundant species in the afforestation of the campus. For Lorenzi (2008), the preference for this species in the composition of roadside trees may be justified by its dense and perennial crown, which provides excellent shading and a deep root system. Therefore, its use is adequate to help mitigate the effect of the heat islands reported in the city of Cuiabá in other studies (Alves \& Biudes, 2011; Santos et al., 2014).

Makhelouf (2009) states that the decreasing temperatures of the parks promote a reduction of the urban heat island, and points to green spaces as being less polluted places in a city. According to the author, green areas have a significant contribution to improving the microclimate and reducing the pollution rate in cities, and denominates these sites as "a city's lungs", being the most important planning sites in the city.

In an interview conducted by Batista et al. (2014), 39\% of people are motivated to move to the UFMT because of the zoo, followed by a $33 \%$ group that seeks the vast green area. Also, campus-goers described a sense of well-being that favors physical and mental health, provided by the wide presence of trees. The environmental perception was also evaluated in the same study, which reported a feeling of silence, satisfaction, tranquility and peace found by the regulars.

Of the total species, $34.7 \%$ produce fleshy fruits, $16.6 \%$ show intense flowering, 3.7\% are palm trees and $42.1 \%$ were in the species group of environmental services. Although they are allochthonous species to the region, Mangifera indica, Genipa americana, Psidium guajava and Anacadium occidentale are trees that produce attractive fleshy fruits to the avifauna of Cuiabá and mainly for the UFMT zoo, which naturally complements their diet and helps in the seed dispersal process. Dipteryx alata, Samanea tubulosa, Guazuma ulmifolia, Caryocar brasiliense, Buchenavia tomentosa, Casearia sylvestris and others are also indigenous species found on the UFMT campus, which contribute to maintaining diversity.

Pivetta \& Silva Filho (2002) indicate that one of the advantages that trees show by their natural characteristics in the urban environment is to provide a better aesthetic effect. The Handroanthus genus comprises about $9.1 \%$ of all campus individuals, and is widely recommended and used in urban tree growing due to the beauty of its flowering. Bauhinia is another genus found on campus with a few specimens; however, it is much used in urban afforestation both for the beauty of its flowers and for its small to medium size, which allows its use in roads under the electricity grid. Palm trees are widely used in the UFMT, where almost all their abundance is concentrated in the central road beds. However, its use is considered inadequate in road forestation and its planting in the urban zone of Cuiabá is prohibited, which includes the UFMT campus (Cuiabá, 2012). Thus, its substitution by woody species is indicated.

At the UFMT, $42.1 \%$ of the species were classified as palm trees, fruit trees or ornamental, which were classified as environmental service providers. Morgenroth et al. (2016) argue that urban trees are important because they regulate the environment in many ways in the city, such as reducing air pollution and storm water management. For Clapp et al. (2014), these environmental services provided by trees in the urban environment depend on their specific characteristics, namely morphological, physiological, temporal, and seasonal or diurnal effects of different species. In this sense, the urban afforestation as a whole must be planned with different species, since each one has its particularities and should be used in the search for environmental balance.

Of the total number of species present on campus, $72.6 \%$ are native to Brazil, $23.7 \%$ originate in other countries and $3.7 \%$ are undetermined due to the non-botanical identification of the species. For the species native to Brazil, Licania tomentosa and Jacaranda cuspidifolia stand out for having the largest number of individuals. Among the species exotic to Brazil, Mangifera indica and Psidium guajava stood out with greater abundance. In addition to the majority of native species to Brazil, $62.6 \%$ of these species occur naturally in the phytogeographic domains of the Cerrado. Despite having an approximate value as required, UFMT afforestation must comprise at least $70 \%$ native species (Cuiabá, 2012). For Farias Castro et al. (2011), the use of autochthonous species in urban afforestation has great potential for both its adaptation to the environment and for preserving biodiversity; both of which are fundamental for balancing the local environment.

According to Riley et al. (2018), the contribution of exotic species to providing ecosystem services should be considered when in appropriate proportions, because, just as with native species, they also perform a number of important benefits in cities, such as carbon sequestration, heat island mitigation, air quality improvement, surface runoff reduction, increased property values, improvement of human health and well-being, and promotion of a greater sense of social connectivity. For Morgenroth et al. (2016), allochthonous species are more likely to positively respond to fluctuating environmental conditions than native species, so it is advantageous to use them.

Based on data from the Instituto Hórus (Instituto Hórus de Desenvolvimento e Conservação Ambiental, 2017), it was found that some exotic species used in the UFMT campus afforestation are characterized by rapid propagation and invasive potential, such as Albizia lebbeck, Leucaena 
leucocephala, Artocarpus heterophyllus, Azadirachta indica, Clitoria fairchildiana, and others. For Ziller (2001), invasive species can have disastrous impacts on the environment and directly affect agricultural, forestry or ornamental activities. In this context, it is necessary to monitor the propagation of these specimens given their rapid development, which may promote greater competition with native species and their possible suppression.

The diversity obtained by the Shannon Index $\left(\mathrm{H}^{\prime}\right)$ was 4.1 , being considered high since it varies from 0 to 5 , in which the higher the value, the greater the diversity. The index is based on the number of species present in a given environment, added to the uniformity between them and their relation to the abundance and dominance degree of each one (Odum, 2012). The calculated value for the Simpson Diversity Index was 0.04 , which expresses high diversity and corroborates the result of the estimated Shannon-Weaver Index. This index expresses the probability that two randomly selected individuals belong to the same species and their value varies from 0 to 1 ; the higher the value, the greater the dominance of certain species; on the other hand, the lower the diversity.

Biondi \& Lima Neto (2011) point out the importance of vegetal species diversification in the urban environment as a source for establishing richer and more diversified fauna. Morgenroth et al. (2016) reiterate the need for species diversity to achieve a stock of resilient trees to contribute to the urban ecosystem stability, where all tree species have desirable and undesirable characteristics, but species selection must be performed strategically to optimize the ecosystem services. Santos et al. (2014) indicate that the green areas in Cuiabá have specific microclimate characteristics, which are different from the non-wooded areas, with a higher formation of humidity islands; a fact that the authors attributed to the consequent decrease in temperature and an increased relative humidity of these places. A similar result was obtained by Alves \& Biudes (2011) in a study on the microclimate on the UFMT campus. However, Marandola Junior et al. (2011) warn about reducing areas for permanent preservation of the streams of Cuiabá; a fact that contributes to an unstable and fragile urban ecosystem and potentiates the risks arising from floods in the capital of Mato Grosso. In this context, the campus green area's contribution to the quality of life of the population regarding mitigation of high temperatures, lowering the low relative humidity and increasing the water flow of the soil of Cuiabá, is corroborated.

The green area of the UFMT is very important for maintaining fauna and flora diversity in the municipality of Cuiabá and can be a reference for planning and implementing other green areas. Many of the local fauna species depend on the food production provided by the fruit species in the campus, as well as the seed dispersion of these vegetal species depending on the fauna displacement for propagating its descendants.

\section{CONCLUSIONS}

The afforestation of the UFMT campus in Cuiabá is rich, with great species diversity in an adequate proportion of Brazilian native species. However, the floristic composition needs adjustment to the native species in the phytogeographic domain of the Cerrado. The floristic structure occasionally needs to be adjusted regarding exotic species with invasive potential, replacing them with autochthonous species. The implanted species in the roadside tree planting must be adapted to the municipal afforestation legislation.

\section{ACKNOWLEDGEMENTS}

To the Coordenação de Aperfeiçoamento de Pessoal de Nível Superior (Capes) for granting a Graduate scholarship to the first author during the study. To UFMT along with PET/Floresta for the opportunity to research and contribute to the knowledge on campus afforestation. To Professor Dr. Diego Tyszka Martinez for the enriching guidance and contribution to the study.

\section{SUBMISSION STATUS}

Received: 29 Mar. 2018

Accepted: 13 Sept. 2018

Associate editor: Rodrigo Studart Corrêa

(D) 0000-0002-9422-2629

\section{CORRESPONDENCE TO \\ Poliane Pierra Patricio Pazini}

Universidade Federal de Mato Grosso (UFMT), Av. Fernando Correa da Costa, 2367, CEP 78060-900, Cuiabá, MT, Brasil

e-mail: polianepatricio@gmail.com

\section{FINANCIAL SUPPORT}

Coordenação de Aperfeiçoamento de Pessoal de Nível Superior (Capes), Grant/Award number: 36000,00.

\section{REFERENCES}

Alencar LD, Cardoso JC. Paisagismo funcional: o uso de projetos que integram mais que ornamentação. Ciência, Tecnologia e Ambiente 2015; 1(1): 1-7.

Alvares CA, Stape JL, Sentelhas PC, Moraes Gonçalves JL, Sparovek G. Köppen's climate classification map for Brazil. Meteorologische Zeitschrift 2013; 22(6): 711-728. 10.1127/0941-2948/2013/0507

Alves EDL, Biudes MS. Os microclimas da Universidade Federal de Mato Grosso/Cuiabá. Raega 2011; 23: 600-620. 10.5380/ raega.v23i0.24923 
Batista BMF, Sousa RAT, Zavala AAZ, Pasa MC, Timofeiczyk Junior R. Percepção ambiental dos frequentadores do campus da Universidade Federal de Mato Grosso. Flovet 2014; 1(6): 98-114.

Biondi D. Floresta urbana: conceitos e terminologias. In: Biondi D. Florestas urbanas. Curitiba: Produção Independente; 2015. p. 11-28.

Biondi D, Lima Neto EM. Introdução de espécies na arborização de ruas. In: Biondi D, Lima Neto EM. Pesquisa em arborização de ruas. Curitiba: Produção Independente, 2011. p. 9-28.

Clapp JC, Ryan III HDP, Harper RW, Bloniarz DV. Rationale for the increased use of conifers as functional green infrastructure: a literature review and synthesis. Arboricultural Journal 2014; 36(3): 161-178. 10.1080/03071375.2014.950861

Cuiabá. Decreto n. 5.144, de 15 de fevereiro de 2012. Dispõe sobre a arborização pública na área urbana e sedes de distrito do município de Cuiabá e dá outras providências. Palácio Alencastro, Cuiabá (2012 Feb. 15).

Eisenlohr PV, Carvalho-Okano RM, Vieira MF, Leone FR, Stringheta ACO. Flora fanerogâmica do campus da Universidade Federal de Viçosa, Viçosa, Minas Gerais. Ceres 2008; 55(4): 317-326.

Farias Castro AS, Moro MF, Rocha FCL. Plantas dos espaços livres da reitoria da Universidade de Fortaleza (Unifor), Ceará, Brasil. Revista Brasileira de Biociências 2011; 9(1): 126-129.

Fundação Coordenação de Projetos, Pesquisas e Estudos Tecnológicos - Coppetec. Flora do Brasil 2020. Rio de Janeiro; c2019 [cited 2017 Jan 25]. Available from: http://bit.ly/2NoEVy0

Instituto Brasileiro de Geografia e Estatística - IBGE. Estimativas da população residente no Brasil e unidades da federação com data de referência em $1^{\circ}$ de julho de 2017. Brasília, DF; 2017.

Instituto Hórus de Desenvolvimento e Conservação Ambiental. Base de dados nacional de espécies exóticas invasoras I3N Brasil. Florianópolis; 2017 [cited 2017 Jan 25]. Available from: http://bit.ly/34g76WG

Kramer JA, Krupek RA. Caracterização florística e ecológica da arborização de praças públicas do município de Guarapuava, PR. Árvore 2012; 36(4): 647-658. 10.1590/S0100-67622012000400007

Loboda CR, Angelis BLD. Áreas verdes públicas urbanas: conceitos, usos e funções. Ambiência 2005; 1(1): 125-139.

Lorenzi H. Árvores brasileiras: manual de identificação e cultivo de plantas arbóreas nativas do Brasil. Nova Odessa: Plantarum, 2008. v. 3.

Makhelouf A. The effect of green spaces on urban climate and pollution. Journal of Environmental Health Science and Engineering 2009; 6(1): 35-40
Marandola Junior E, D’Antona AO, Ojima R. População, ambiente e desenvolvimento: mudanças climáticas e urbanização no centrooeste. Campinas: Núcleo de Estudos da População/Unicamp; 2011.

McHale MR, McPherson EG, Burke IC. The potential of urban tree plantings to be cost effective in carbon credit markets. Urban Forestry and Urban Greening 2007; 6: 49-60. 10.1016/j.ufug.2007.01.001

Milano MS, Dalcin EC. Arborização de vias públicas. Rio de Janeiro: Light; 2000.

Missouri Botanical Garden. Tropicos. Saint Louis; 2016 [cited 2017 Jan 1]. Available from: http://bit.ly/36f9TkG

Morgenroth J, Östberg J, van den Bosh CK, Nielsen AB, Hauer R, Sjöman $\mathrm{H}$ et al. Urban tree diversity: taking stock and looking ahead. Urban Forestry and Urban Greening 2016, 15: 1-5. 10.1016/j.ufug.2015.11.003

Odum EP. Ecologia. Rio de Janeiro: Guanabara Koogan; 2012.

Pinheiro CR, Souza DD. A importância da arborização nas cidades e sua influência no microclima. Gestão e Sustentabilidade Ambiental 2017; 6(1): 67-82. 10.19177/rgsa.v6e1201767-82

Pivetta KFL, Silva Filho DF. Arborização urbana. Jaboticabal: Unesp/FCAV/Funep; 2002. (Boletim Acadêmico: Série Arborização Urbana). $74 \mathrm{p}$.

R Development Core Team. R: A language and environment for statistical computing. Vienna: R Foundation for Statistical Computing; 2015.

Riley CB, Herms DA, Gardiner MM. Exotic trees contribute to urban forest diversity and ecosystem services in inner-city Cleveland, OH. Urban Forestry and Urban Greening 2018; 29: 367-376. 10.1016/j.ufug.2017.01.004

Santos HG, Jacomine PKT, Anjos LHC, Oliveira VA, Lumbreras JF, Coelho MR et al. Sistema brasileiro de classificação de solos. 3rd ed. Brasília, DF: Embrapa Solos, 2013.

Santos FMM, Oliveira AS, Nogueira MCJA, Musis CJ, Nogueira JS. Análise do clima urbano de Cuiabá, MT, Brasil por meio de transectos móveis. Paranoá 2014; (11): 45-53. 10.18830/issn.16790944.n11.2014.12083

Stevens PF. Angiosperm Phylogeny Website, 2016 [cited 2016 Sept. 10]. Available from: http://bit.ly/2N4oU1e

Ziller SR. Plantas exóticas invasoras: a ameaça da contaminação biológica. Ciência Hoje 2001; (178): 77-79. 